\title{
Ouvindo Augusto - dados para uma discomusicografia de Augusto de Campos
}

Marcelo Dolabela I Poeta, Jornalisa e Ensaísta

Resumo: O texto que se segue contém uma relação cronológica de trabalhos de Augusto de Campos relacionados à música, incluindo texto de apresentação de discos, poemas gravados do elou pelo poeta, poemas visuais reproduzidos em capas de discos, versões de letras gravadas ou por Augusto ou por outros intérpretes.

Palavras-chave: Augusto de Campos, música, poética de invenção.

Caetano Veloso, na canção "Saudosismo": "A realidade é que / aprendemos com João / pra sempre / a ser desafinados / ser desafinados / ser desafinados / ser...". Augusto de Campos, em lance de dados semelhante, "nos ensinou a 'ouviver' a música com outra afinação".

Aprendizado multivocoverbivisual: teoria \& crítica \& invenção \& prosa porosa \& poesia \& popcretos \& profilograma \& melodiadetimbres \& performance \& produção \& edição \& tradução \& intradução \& transcriação \& gravação \& midiacidade / city / cité.

Esta breve cronomusicodiscografia repete o gesto Augusto em relação à obra de Lupicínio Rodrigues - "Dados para uma discografia de 
O eixo e a roda: $v .13,2006$

Disponivel em: http://www.letras.ufmg.br/poslit

Lupicínio Rodrigues" (In: Balanço da bossa e outras bossas. 2 $2^{\underline{a}}$ ed. São Paulo: Perspectiva, 1974) - e homenageia, nos 50 anos da poesia concreta, Augusto e a sua poética de invenção.

1968 - Texto-colagem-manifesto: Tropicália: panis et circensis. LP Tropicália: panis et circensis. Philips. Fechando o texto (um fragmento do artigo "João Gilberto e os jovens baianos", de Augusto de Campos. In: Correio da Manhã. 18/08/1968): "IMÓVEL. JOÃO GILBERTO E AUGUSTO DE CAMPOS SENTADOS. / AUGUSTO - E o que é que eu digo a eles? / JOÃO - Diga que eu estou daqui olhando pra eles".

1970 - "Novos Baianos depois do dilúvio". Texto: Augusto de Campos. In: NOVOS BAIANOS. LP É ferro na boneca!. RGE.

1972 - "Cidade". Poema: Augusto de Campos. Faixa: "Senhor Cidadão", de Tom Zé. In: TOM ZÉ. LP Tom Zé. Continental. Relançamento em CD: Clássicos da MPB - Série Dois Momentos - Se o caso é chorar / Todos os olhos. Continental / EastWest, 2000.

1972 - "Só somente só". Texto: Augusto de Campos. In: NOVOS BAIANOS. LP Acabou chorare. Som Livre.

1973 - "Cademar". De: Tom Zé \& Augusto de Campos. In: TOM ZÉ. LP Todos os olhos. Continental. Relançamentos: LP Nova história da Música Popular Brasileira - Hermeto Paschoal, Djalma Corrêa, Walter Franco, Tom Zé. Abril Cultural, 1979. LP Massive hits - The best of Tom Zé - O melhor de Tom Zé. Luaka Bop/BMG Ariola Discos, 1990. CD: Clássicos da MPB - Série Dois Momentos - Se o caso é chorar / Todos os olhos. Continental / EastWest, 2000.

1973 - "eu e a Bahia estamos sempre nos encontrando...". Texto: Augusto de Campos. In: TOM ZÉ. LP Todos os olhos. Continental. Relançamento em CD: Clássicos da MPB - Série Dois Momentos - Se o caso é chorar / Todos os olhos. Continental / EastWest, 2000.

1973 - "Olho por olho". Poema: Augusto de Campos. Na capa do LP Todos os olhos, de Tom Zé, Continental, 1973. Relançamento em CD (fragmento): Clássicos da MPB - Série Dois Momentos - Se o caso é chorar / Todos os olhos. Continental / EastWest, 2000. 
Belo Horizonte, p. 1-232

Disponivel em: http://www.letras.ufmg.br/poslit

1973 - "Soneterapia". Soneto de Augusto de Campos. In: Navilouca. Na primeira estrofe: "drummond perdeu a pedra: é drummundano / joão cabral entrou pra academia / custou mas descobriram que Caetano / era o poeta (como eu já dizia)".

1976 - "Lygia Fingers". Poema: Augusto de Campos. Música: Marcus Vinicius. In: MARCUS VINICIUS. LP Trem dos condenados.

Discos Marcus Pereira.

1976 - "Desmadamentos". Decálogo-manifesto de: Caetano Veloso. In: Música do Planeta Terra, n.5. No "desmandamento 4": "O 'Pulsar' de Augusto de Campos é o melhor poema impresso da era do rock".

1976 - "Gil contando o que Dominguinhos disse...". Texto de Caetano Veloso. In: Ta-ta-ta: "Eu cheio de preguiça, dentro da violência do mundo. No gravador, a voz linda do poeta Augusto de Campos, cantando o samba do Sr. Eurico (de Campos - pai de Augusto). O poeta Waly Salomão 76 comenta a semelhança do canto de Augusto com o de Paulinho da Viola. A vida entre músicos. Augusto me disse uma vez que era um camicase. O radicalismo da viagem literária em que ele e seus amigos se meteram levou seu nome ao fogo das batalhas de uma guerra de beleza sem razão. A doçura impecável de sua voz me faz agora entrar em contato com a solidão do guerreiro, a sua felicidade escondida com uma saudade escondida, voz de alguém que só canta assim porque nunca canta assim a não ser quando o faz, alguém que está em outra, completamente nesta, alguém que está em outra dimensão. A peça é idêntica a um perfeito 'antique' - o piano e o samba do Sr. Eurico, Augusto cantando com uma voz aguda cristalina afinadíssima como alguns cantores dos anos 30 - e, no entanto, não parece como nada. Dever ser um grande momento íntimo de um grande poeta. E ele que me considera um poeta". O texto pode ser lido, nas obras de Caetano Veloso: Alegria, alegria - uma Caetanave organizada por Waly Salomão. Pedra Q Ronca. Caetano Veloso - O mundo não é chato. Organização: Eucanaã Ferraz. Companhia das Letras, 2005.

1977 - "Louca me chamam" ("Crazy he call's me"). De: C. Sigman \& B. Russel. Versão: Augusto de Campos. In: GAL COSTA. LP Caras \& bocas. Philips. / GAL COSTA. LP Gal Costa - Meu bem meu mal. Fontana. Coletânea. 
1977 - "Solitude". De: Duke Ellington \& E. de Lange. Versão: Augusto de Campos. In: GAL COSTA. LP Caras \& bocas. Philips.

1978 - "Berceuse dos elefantes". De e com: Walter Franco. Com a dedicatória: "A Augusto de Campos e Lígia”. In: LP Respire fundo. CBS Epic.

1978 - "Sampa". De e com: Caetano Veloso. In: LP Muito. Philips. Primeira estrofe: "Alguma coisa acontece no meu coração / que só quando cruza a Ipiranga e a avenida São João / é que quando eu cheguei por aqui eu nada entendi / da dura poesia concreta de tuas esquinas...".

1979 - "Dias, dias, dias (de Poetamenos)". Poema: Augusto de Campos. Música: Caetano Veloso. Música incidental: "Volta", de Lupicínio Rodrigues. In: CAETANO VELOSO. CS "Dias, dias, dias" / "Pulsar". Philips, 1979. Encartado no livro: CAMPOS, Augusto de. Viva vaia - Poesia 1949-1979. Livraria Duas Cidades. Há registro que a faixa foi incluída em uma coletânea de Caetano Veloso, lançada no e para o mercado japonês.

1979 - "O pulsar". Poema: Augusto de Campos. Música: Caetano Veloso. In: CAETANO VELOSO. CS "Dias, dias, dias" / "Pulsar". Philips, 1979. Encartado no livro: CAMPOS, Augusto de. Viva vaia - Poesia 19491979. Livraria Duas Cidades.

1979 - "Elegia". De: Péricles Cavalcanti \& Augusto de Campos. A partir de poema de John Donne, século XVII. In: CAETANO VELOSO. LP Cinema transcendental. PolyGram.

1980 - "Mamãe merece" ("God bless the child"). De: Herzog Jr. \& Billie Holiday. Versão: Augusto de Campos \& Rogério Duarte. In: ZIZI POSSI. LP Zizi Possi. PolyGram.

1982 - "Asa linda (Little wing)". De: Jimi Hendrix. Versão: Augusto de Campos. In: TIAGO ARARIPE. LP Cabelos de Sansão.

1982 - "Jaguadarte". De: Arrigo Barnabé \& Augusto de Campos. recriação de Augusto de Campos, de um trecho do poema "Jabberworcky" de Lewis Carroll. In: TETÊ ESPÍNDOLA. LP Pássaros na garganta. Som da Gente. 
Belo Horizonte, p. 1-232

Disponivel em: http://www.letras.ufmg.br/poslit

1982 - "Voz de pássaros e de rio...". Texto: Augusto de Campos. In: TETÊ ESPÍNDOLA. LP Pássaros na garganta. Som da Gente.

1982 - "Cinzas, negras borboletas" ("Pierrot Lunaire"). De: Arnold Schoenberg. Texto original: Hartleben. Tradução: Augusto de Campos. In: ELIETE NEGREIROS. CD Outros sons. Vôo Livre / EMI Odeon.

1983 - "Românticos a go-go". Composição de: Júlio Barrroso. In: GANG 90 \& ABSURDETTES. LP Essa tal de Gang 90. RCA. Letra-onomástica. No sétimo verso: Cummings, John Donne, Augusto, Pagu.

1984 - "Noigandres". Poema: Arnaut Daniel. Tradução: Augusto de Campos. Música: Vitor Ramil. In: VITOR RAMIL. LP A paixão de V segundo ele próprio. Som Livre. Primeira estrofe do poema "Er vei vermeills, vertz, blaus, blancs, gruocs" ("Vermelho e verde e branco e blau").

1984 - "O pulsar". Poema: Augusto de Campos. Música: Caetano Veloso. In: CAETANO VELOSO. LP Velô. Philips.

1984 - "Som sem som". Poema concreto de Augusto de Campos. Música: Gilberto Mendes. In: MADRIGAL RENASCENTISTA. LP Música Nova do Brasil. Promus - Projeto Memória Musical Brasileira / Funarte. Regente: Afrânio Lacerda.

1984 - "Num barraco precário". Fragmento do "Mauberley", de: Ezra Pound. Tradução: Augusto de Campos. Música: Passoca. In: PASSOCA. LP Sonora garoa. Barclay.

1985 - "Sophisticated lady". De: Duke Ellington. Versão: Augusto de Campos. In: ELZA SOARES. LP Elza Soares. Com a participação especial de Caetano Veloso. LP Somos todos iguais. Som Livre. Há registro de uma gravação da cantora Neusa Pinheiro.

1986 - "Além do arco-íris" ("Over the rainbow"). De: H. Arlen \& E. Y. Harburg. Versão: Augusto de Campos. In: VÂNIA BASTOS. LP Vânia Bastos. Copacabana.

1986 - "Shirley Sombra". De: Arrigo Barnabé. Recriação de Augusto de Campos de versão inglesa de Edward Fitzgerald do Rubayat de Omar Khayyam. 
O eixo e a roda: $v .13,2006$

Disponivel em: http://www.letras.ufmg.br/poslit

Gravação: Arrigo Barnabé. Participação especial: Ana Amélia e Sossega Leão. In: ARRIGO BARNABÉ. LP Cidade oculta (trilha sonora do filme). Barclay.

1986 - "Tudo está dito". Poema: Augusto de Campos. Música: Arrigo Barnabé. In: ELIETE NEGREIROS. LP Ângulos. Copacabana / Bom Tempo.

1989 - "Nas rochas". Poema: Vielimir Khlébnikov. Versão: Augusto de Campos. Música: Paulo Ricardo \& Fernando Deluqui. In: PAULO RICARDO. LP Paulo Ricardo. CBS.

1990 - "O pulsar". Poema: Augusto de Campos. Música: Caetano Veloso. In: CAETANO VElOSO. LP Caetano Veloso. Philips.

1991 - "Elegia". De: Péricles Cavalcanti \& Augusto de Campos. A partir de poema de John Donne, século XVII. In: PÉRICLES CAVALCANTI. LP Canções, PolyGram.

1991 - "Nuvoleta". De: Péricles Cavalcanti \& Augusto de Campos. A partir de um fragmento do "Finnegans Wake", de James Joyce. In: PÉRICLES CAVALCANTI. LP Canções, PolyGram.

1991 - "O verme e a estrela". Poema: Pedro Kilkerry. Música: Cid Campos. Gravação: Cid Campos. Leitura: Augusto de Campos. In: LP Rock de autor. Manifesto.

1992 - "Canto I do Inferno". Poema: Dante Alighieri. Versão: Augusto de Campos. Música: Arrigo Barnabé - Mané Silveira \& Paulo Braga. In: ARRIGO BARNABÉ. CD Façanhas. Camerati. As 20 primeiras estrofes do canto.

1994 - "De ninar". De: Cid Campos \& Augusto de Campos. In: Canções de ninar - 22 canções inéditas para embalar seu filho. Coleção Palavra cantada. Produção: Sandra Peres \& Paulo Tatit. Produtor associado: Manny Monteiro.

1994 - "Mensagem". Poema: John Donne. Tradução: Augusto de Campos. Música: Péricles Cavalcanti. In: TETÊ ESPÍNDOLA. CD Só Tetê. Camerati.

1994 - "O verme e a estrela". Poema: Pedro Kilkerry. Música: Cid Campos. Gravação: Adriana Calcanhoto. Participação especial: Augusto de Campos. In: ADRIANA CALCANHOTO. LP A fábrica do poema. CBS. 
1994 - Poesia é risco. CD: Augusto de Campos \& Cid Campos. Mercury. Faixas: "O rei menos o reino"; "Lygia Fingers"; "Bestiário"; "Tensão"; "Corsom"; "Caracol"; "Cidade/city/cité"; "Tudo está dito"; "O quasar"; "SOS"; "Risco"; "Poemabomba"; "TVgrama 1 / Tombeau de Mallarmé"; "TVgrama 2 / Anttenae of the race"; "Cançãonoturnadabaleia"; "Viventes e vampiros"; "O barco bêbado" (Arthur Rimbaud - versão (recriação) de Augusto de Campos); "O verme e a estrela" (Pedro Kilkerry); "Vogais (Arthur Rimbaud - versão (recriação) de Augusto de Campos); "A estrela chorou rosa..." (Arthur Rimbaud - versão (recriação) de Augusto de Campos); "Os corvos" (Arthur Rimbaud - versão (recriação) de Augusto de Campos); "Canção da mais alta torre" (Arthur Rimbaud - versão (recriação) de Augusto de Campos); "Cocheiro bêbado" (Arthur Rimbaud - versão (recriação) de Augusto de Campos); "Soneterapia 2"; "O tygre" (William Blake - versão (recriação) de Augusto de Campos); "do 'Finnegans wake': a queda” (James Joyce - versão (recriação) de Augusto de Campos); "do 'Finnegans wake': colidouescapo" (James Joyce - versão (recriação) de Augusto de Campos); "Pós-tudo"; "Chegou a noite" (Eurico de Campos).

1995 - "Toda vez que eu digo adeus" ("Every time we say goodbye"). Música e letra: Cole Porter. Versão: Augusto de Campos. Gravação: Cauby Peixoto \& Ney Matogrosso. In: CAUBY PEIXOTO. CD Cauby canta Sinatra. Som Livre.

1995 - "O pulsar". Poema: Augusto de Campos. Música: Caetano Veloso. In: CAETANo Veloso. Fina estampa ao vivo. PolyGram, 1995. Relançamento: série Millennium.

1995 - "Elegia". De: Péricles Cavalcanti \& Augusto de Campos. A partir de poema de John Donne, século XVII. In: SIMONE. CD Simone Bittencourt de Oliveira. Sony Music.

1996 - "Êxtases". Poema: Augusto de Campos. Música: Péricles Cavalcanti \& Cid Campos. Gravação: Péricles Cavalcanti. In: Mil e uma (trilha sonora). Natasha Records.

1996 - "Longe e perto". Poema: Augusto de Campos. Música: Péricles Cavalcanti. Gravação: Péricles Cavalcanti. In: Mil e uma (trilha sonora). Natasha Records. 
O eixo e a roda: $v .13,2006$

Disponivel em: http://www.letras.ufmg.br/poslit

1996 - "Poema cauda". Poema: Lewis Carroll. Tradução: Augusto de Campos. Música: Péricles Cavalcanti. Gravação: Péricles Cavalcanti. In: Mil e uma (trilha sonora). Natasha Records.

1996 - "Fractal". De: Ruriá Duprat. Introdução: "Pós tudo", de Augusto de Campos, extraído do CD Poesia é risco, de Augusto de Campos \& Cid Campos, voz: Augusto de Campos. Gravação: Ruriá Duprat. In: CD Mundo São Paulo 50 anos PUC-SP SESC-SP. Pau Brasil.

1997 - "A sopa”. Poema: Lewis Carroll. Versão: Augusto de Campos. Música: Marcos Pimenta. In: MARIANTIVEL. CD O Grande Ah!....

1998 - "Com som sem som (Tensão)". Poema: Augusto de Campos. Música: Eduardo Guimarães Álvares. In: CD Poesia paulista - 12 canções. Secretaria da Cultura de São Paulo. Direção e coordenação: Dante Pignatari.

1998 - "Passa da uma". Poema: Vladímir Maiakóvski. Versão: Augusto de Campos. Música: Beatriz Azevedo. In: BEATRIZ AZEVEDO. CD Bum bum do poeta. Natasha Records.

1999 - Ouvindo Oswald. CD Ouvindo Oswald - a poesia de Oswald de Andrade. Funarte/Itaú Cultural. Augusto de Campos lê: "Noturno"; "3 de maio"; "Ditirambo"; "Cielo e maré"; "Longo da linha" e "Procissão do enterro"; Haroldo de Campos, Décio Pignataria e Augusto de Campos lêem: de O escaravelho de ouro: "Promontório"; "Epitáfio no 1"; "Epitáfio no 2" e "Plebiscito".

1999 - "Orelha para Oswald". Texto: Augusto de Campos. In: CD Ouvindo Oswald - a poesia de Oswald de Andrade. Funarte/Itaú Cultural.

2000 - "Você é o meu" ("You're the top"). De: Cole Porter. Versão: Augusto de Campos. Gravação: Tom Zé. In: Canções, versões. Geléia Geral.

2000 - "Walter Franco". Texto: Augusto de Campos. In: WALTER FRANCO. CD: Clássicos da MPB - Série Dois Momentos - Revolver / Ou não. Continental / EastWest.

2000 - Viva vaia - poesia 1949-1949. Livro-CD de Augusto de Campos. Ateliê Editorial. Faixas do CD: "O rei menos o reino - 1"; "Lygia Fingers"; "Bestiário"; "Salto" (faixa inédita); "Ovonovelo" (faixa inédita); "Tensão"; "Uma vez" (faixa inédita); "Corsom"; "Caracol"; "Cidade/city/cite"; "O anti- 
ruído" (faixa inédita); "Rever" (faixa inédita); "O tygre"; "O quasar"; "Tudo está dito". Demais faixas, extraídas do CD Poesia é risco, de Augusto de Campos \& Cid Campos. Mercury, 1994.

2001 - "Êxtases". Poema: Augusto de Campos. Música: Cid Campos \& Péricles Cavalcanti. In: CID CAMPOS. CD No lago do olho. Dabliú Discos.

2001 - "Flor da boca". Poema: Augusto de Campos. Música: Cid Campos. In: CID CAMPOS. CD No lago do olho. Dabliú Discos. Gaita: Augusto de Campos.

2001 - "Tempoespaço". Poema: Augusto de Campos. Música: Cid Campos. In: CID CAMPOS. CD No lago do olho. Dabliú Discos. Voz (poema): Augusto de Campos.

2001 - "Velocidade". Poema: Ronaldo Azeredo. Música: Cid Campos. In: CID CAMPOS. CD No lago do olho. Dabliú Discos. Voz (poema): Augusto de Campos.

2001 - "Viventes e vampiros". Poema: Augusto de Campos. Música: Cid Campos. In: CID CAMPOS. CD No lago do olho. Dabliú Discos.

2001 - "Balalaica". Poema: Vladímir Maiakóvski. Tradução: Augusto de Campos. Música: Alceu Valença. In: Forró lunar. Sony Music.

2002 - "Nalgum lugar". Poema: e. e. cummings. Tradução: Augusto de Campos. Música: Zeca Baleiro. In: ZECA BALEIRO. CD Líricas. MZA Music.

2002 - "Intradução". Música: Walter Franco. Letra: poema de Augusto de Campos, adaptado do texto do trovador Bernart de Ventandorn. In: WALTER FRANCO. CD Tutano. YB Music.

2002 - "Elegia". De: Péricles Cavalcanti \& Augusto de Campos. A partir de poema de John Donne, século XVII. In: ANA CRISTINA. CD Poemas musicados ao vivo.

2002 - "Pulsar" / "Quasar". Poemas: Augusto de Campos. Composição musical: "L'alibi della parola" (1994), de Salvatore Sciarrino. In: NEUE VOCALSOLISTEN STUTTGART. CD Fuoco e Giacchio (Madrigale). Stradivarius. Made in Itália. 
2002 - Arte concreta paulista - Grupo Noigandres. Livro-CD. Curadores: Lenora de Barros \& João Bandeira. Cosac \& Naify / Centro Universitário Maria Antonia da USP. Poemas de e interpretados por Augusto de Campos incluídos no CD: "O rei menos o reino"; "Por suposto (do Poetamenos)"; "Lygia Fingers (do Poetamenos)"; "Nossos dias com cimento (do Poetamenos)"; "Eis os amantes (do Poetamenos)"; "Salto"; "Bestiário"; "Ovonovelo"; "Tensão" - c/ José Lino Grünewald \& Ecila Grünewald; "Uma vez" - c/ José Lino Grünewald \& Ecila Grünewald; "Eixo"; "Sem um número"; "Sangueareia" - c/ José Lino Grünewald; "Caracol" - c/ Lygia Campos; "Greve" - c/ José Lino Grünewald \& Ecila Grünewald; "Plusvalia" - c/ Haroldo de Campos \& José Lino Grünewald; "Cidade". Poemas de outros poetas com a participação de Augusto de Campos, como intérprete: "Música de coreto da minha autoria na oportunidade das exéquias de um venéreo ancião (de Bufoneria brasiliensis)", de Décio Pignatari - intérpretes: Haroldo de Campos, Augusto de Campos (coro), Lygia Campos (coro), José Lino Grünewald (coro) e Ecila Grünewald (coro); "Cristal", de Haroldo de Campos, intérpretes: Augusto de Campos \& Ecila Grünewald; "Cesse", de José Lino Grünewald - intérpretes: José Lino Grünewald \& Augusto de Campos; "Rio raio", de José Lino Grünewald - intérpretes: José Lino Grünewald \& Augusto de Campos; "Petróleo", de José Lino Grünewald - intérpretes: José Lino Grünewald, Ecila Grünewald \& Augusto de Campos; "Forma", de José Lino Grünewald - intérpretes: José Lino Grünewald \& Augusto de Campos; "Revolução", de José Lino Grünewald - intérpretes: José Lino Grünewald \& Augusto de Campos; "Velocidade", de Ronaldo Azeredo - intérpretes: José Lino Grünewald \& Augusto de Campos.

2003 - "Ão". Poema: Augusto de Campos. Música: Aldo Brizzi. Canto: Caetano Veloso. Leitura do poema: Augusto de Campos. CD Brizzi do Brasil. Eldorado. Edição européia: Amianta Record, 2004.

2004 - "Ão". Poema: Augusto de Campos. Música: Aldo Brizzi. In: AÇO DE AÇÚCAR. CD Aço de açúcar. NovoDisc Mídia Digital. Vocal: Graça Reis.

2004 - "Beijo de amor celeste". Música: Cid Campos. Poema: Quirinus Kuklmann - séc. XVII - tradução: Augusto de Campos. In: CID CAMPOS. CD Fala da palavra. 
Belo Horizonte, p. 1-232

Disponível em: http://www.letras.ufmg.br/poslit

2004 - "O pulso das palavras". Poema: Vladímir Maiakóvski. Tradução: Augusto de Campos. Música: Cid Campos. In: CID CAMPOS. CD Fala da palavra.

2004 - "O que quer que". Poema: Augusto de Campos. Música: Cid Campos. In: CID CAMPOS. CD Fala da palavra.

2004 - "Sem saída". Poema: Augusto de Campos. Música: Cid Campos. In: CID CAMPOS. CD Fala da palavra.

2005 - "O mocho e a gatinha" ("The owl and the pussy-cat"). Poema: Edward Lear. Tradução: Augusto de Campos. Música: Cid Campos. In: ADRIANA PARTIMPIM. CD Adriana Partimpim. Sony \& BMG.

2005 - "O mocho e a gatinha" ("The owl and the pussy-cat"). Poema: Edward Lear. Tradução: Augusto de Campos. Música: Cid Campos. In: ADRIANA PARTIMPIM. DVD Adriana Partimpim - o show. Sony \& BMG.

2005 - "Canção da falsa tartaruga" ("The Mock Turtle"s story"). Poema: Lewis Carroll - do livro Alice no País das Maravilhas. Cap. IX, "A história da Falsa Tartaruga”, Tradução: Augusto de Campos. Música: Cid Campos. In: ADRIANA PARTIMPIM. DVD Adriana Partimpim - o show. Sony \& BMG.

Abstract: The following text enlists in chronological order the work of Augusto de Campos in the field of music and recording, including presentations of records, his poems recorded either by himself or others, visual poems printed on albums' jackets, Portuguese versions of poems and lyrics made by Campos and recorded by a number of performers. Key words: Augusto de Campos, music, poetics of invention. 